\title{
UTILIDAD DE LA CITOMETRÍA DE FLUJO PARA LA DETECCIÓN DE Pseudomonas aeruginosa PRODUCTORAS DE METALOBETALACTAMASAS
}

\author{
Iván Alexander Salinas-Salcedo@1,2,a,b, José Antonio Saldaña-Jiménez@2, 2,,d, \\ José Manuel Guevara-Canales [3,a, Edgar Gonzales-Escalante ${ }^{[4,5, a, e}$ \\ ${ }^{1}$ Hospital de Alta Complejidad Virgen de la Puerta, EsSalud, La Libertad, Perú. \\ ${ }^{2}$ Universidad Nacional de Trujillo, La Libertad, Perú. \\ ${ }^{3}$ Becton Dickinson, Perú. \\ ${ }^{4}$ Centro de Investigaciones Tecnológicas, Biomédicas y Medioambientales (CITBM), Universidad Nacional Mayor de San \\ Marcos, Lima, Perú. \\ ${ }^{5}$ Laboratorio de Resistencia Bacteriana, Facultad de Farmacia y Bioquímica, Instituto de Investigaciones en Bacteriología y \\ Virología Molecular (IBaViM), Universidad de Buenos Aires Argentina. \\ a Tecnólogo médico; ${ }^{b}$ magister en Ciencias con mención en Microbiología Clínica; ${ }^{c}$ magister en Biotecnología y Bioenergía; \\ ${ }^{\mathrm{d}}$ doctor en Ciencias Biológicas; ${ }^{\mathrm{e}}$ magister en Microbiología. \\ * El artículo es parte de la tesis del autor Iván Alexander Salinas Salcedo para optar al grado académico de magister en \\ Ciencias con mención en Microbiología Clínica por la Universidad Nacional de Trujillo; 2019.
}

\section{RESUMEN}

Con el objetivo de determinar la utilidad de la citometría de flujo para la detección de Pseudomonas aeruginosa productoras de metalobetalactamasas (MBL), se estudiaron aislamientos de $P$. aeruginosa genotípicamente caracterizados del cepario del laboratorio de Epidemiología Molecular y Genética de la Universidad Nacional Mayor de San Marcos. Se analizaron 29 aislamientos (17 productoras de MBL y 12 no productoras de MBL) con el kit de viabilidad celular FACSCalibur (Becton Dickinson). Se utilizaron dos tratamientos, uno con meropenem y el otro con meropenem-EDTA. Usando la razón de aumento de fluorescencia en las células no vivas, se demostró una diferencia significativa entre las productoras de MBL y las no MBL, considerando como punto de corte una razón $>1,6$. Se determinó una sensibilidad de $94,1 \%$ y una especificidad del $100 \%$. La citometría de flujo constituye una alternativa para la detección de $P$. aeruginosa productora de MBL.

Palabras clave: Pseudomonas aeruginosa; Carbapenémicos; Beta-Lactamasas; Resistencia Betalactámica; Citometría de Flujo (Fuente: DeCS BIREME).

\section{UTILITY OF FLOW CITOMETRY FOR DETECTING METALLO-BETA-LACTAMASE-PRODUCING Pseudomonas aeruginosa}

\section{ABSTRACT}

In order to determine the utility of flow cytometry for detecting metallo-beta-lactamase (MBL)-producing Pseudomonas aeruginosa, we used genotypically characterized $P$. aeruginosa isolates from the Molecular Epidemiology and Genetics Laboratory of the Universidad Nacional Mayor de San Marcos. A total of 29 isolates (17 MBL-producing and 12 non-MBL-producing) were analyzed with the FACSCali-

Citar como: Salinas-Salcedo IA, Saldaña-Jiménez JA, Guevara-Canales JM, Gonzales-Escalante E. Utilidad de la citometría de flujo para la detección de Pseudomonas aeruginosa productoras de metalobetalactamasas. Rev Peru Med Exp Salud Publica. 2020;37(4):7004. doi: https://doi.org/10.17843/ rpmesp.2020.374.4825.

Correspondencia: Edgar Gonzales Escalante; egones_5@hotmail.com bur (Becton Dickinson) cell viability kit. Two treatments were used, one with meropenem and the other with meropenem-EDTA. A significant difference between MBL and non-MBL-producing $P$. aeruginosa was demonstrated using the fluorescence ratio in non-living cells, considering a cut-off point of $>1.6$. We determined a sensitivity of $94.1 \%$ and a specificity of $100 \%$. Flow cytometry represents an alternative for the detection of MBL-producing P. aeruginosa.

Keywords: Pseudomonas aeruginosa; Carbapenems; beta-Lactamases; beta-Lactam resistance, Flow Cytometry (Source: MeSH NLM).

\section{INTRODUCCIÓN}

Pseudomonas aeruginosa es uno de los principales patógenos oportunistas causante de una amplia variedad de infecciones nosocomiales, como sepsis, neumonía, infecciones del tracto 
urinario y de las partes blandas. Además, puede presentar resistencia por mecanismos intrínsecos o adquiridos, a varios tipos de antibióticos, como los betalactámicos, aminoglucósidos, fluoroquinolonas y polimixinas, por ello el tratamiento de infecciones por $P$. aeruginosa se limita a ciertas clases de antibióticos, entre ellas los carbapenémicos, siendo estos considerados los betalactámicos más potentes contra este microorganismo ${ }^{(1)}$.

Los carbapenémicos, como el imipenem (IPM) y el meropenem (MEM), son los antibióticos de mayor eficacia para el tratamiento de las infecciones causadas por bacterias gramnegativas multidrogorresistentes (MDR), como la $P$. aeruginosa $^{(2)}$. La resistencia a carbapenémicos se puede dividir en dos tipos de mecanismos: el no productor de carbapenemasa (No-PC) y el productor de carbapenemasa (PC). El primero posee sensibilidad reducida a los carbapenémicos debido a la sobreexpresión de betalactamasas tipo AmpC, pérdida de porinas (OprD) o bombas de flujo ${ }^{(3)}$. En contraste, el PC adquiere un gen transmisible que produce una enzima específica para la hidrólisis de los carbapenémicos, una carbapenemasa ${ }^{(4)}$.

La producción de carbapenemasas es uno de los más importantes mecanismos por el cual $P$. aeruginosa se vuelve resistente a los carbapenémicos mediante elementos genéticos móviles. Estas enzimas son betalactamasas, que además de hidrolizar carbapenémicos, lo hacen prácticamente con todos los betalactámicos. Sobre la base de la clasificación molecular de Ambler, las carbapenemasas se dividen en serinobetalactamasas $(\mathrm{SBL})$ de clase $\mathrm{A}$, metalobetalactamasas (MBL) de clase B y SBL de clase D. Mientras que las SBL requieren un resto de serina en su sitio activo, las MBL requieren cationes metálicos divalentes como el zinc, para la actividad enzimática ${ }^{(1,5)}$.

En la práctica clínica se considera esencial la detección rápida y exacta de $P$. aeruginosa $\mathrm{PC}$; y de esta manera implementar el tratamiento oportuno y específico para el control de la infección ${ }^{(9)}$. Sin embargo, esto toma un promedio de 55 horas entre la recolección para el cultivo y la entrega del reporte del antibiograma; se debe tener en cuenta que la mortalidad aumenta en $7,6 \%$ por cada hora de retraso en la administración del tratamiento efectivo ${ }^{(6)}$.

En el Perú, la frecuencia de MBL en $P$. aeruginosa resistente a los carbapenémicos ha aumentado en la última década, variando de $15,7 \%$ en $2011^{(7)}$ a $31,6 \%$ en $2016^{(8)}$, lo que demuestra que este mecanismo de resistencia está presente en nuestro medio y es importante su detección rápida y certera por parte de los laboratorios de microbiología clínica.

Se han propuesto varias pruebas fenotípicas basadas en el uso de inhibidores para el tamizaje de $P$. aeruginosa productoras de MBL, sin embargo, todas ellas deben confirmarse mediante técnicas de biología molecular para la identificación del gen. Los métodos fenotípicos utilizan

\section{MENSAJES CLAVE}

Motivación para realizar el estudio: Pseudomonas aeruginosa es una bacteria oportunista causante de infecciones nosocomiales donde la resistencia a carbapenémicos es cada vez mayor. Presentamos un método de citometría de flujo (CF) para la detección de metalobetalactamasas (MBL).

Principales hallazgos: Con el método de CF para la detección de MBL se obtuvo una sensibilidad de $94,1 \%$ y una especificidad del $100 \%$; además, un valor predictivo positivo de $100 \%$.

Implicancias: Los ensayos de CF podrían proporcionar un método rápido y de fácil interpretación para detectar $P$. aeruginosa productora de MBL.

quelantes de metal, como el ácido etilendiaminotetraacético (EDTA) o ácido dipicolínico (DPA) ${ }^{(9)}$.

La citometría de flujo (CF) es una tecnología de análisis cuantitativo la cual permite caracterizar poblaciones celulares a un nivel individual, las células son iluminadas por una lámpara láser, obteniendo intensidades de señal detectadas por un sensor de fluorescencias, para finalmente correlacionarlas con parámetros estructurales o funcionales de la célula analizada para su clasificación ${ }^{(10)}$. El objetivo de este estudio fue evaluar la utilidad de la CF para la detección de $P$. aeruginosa productoras de MBL.

\section{EL ESTUDIO}

Se realizó un estudio descriptivo de aislamientos clínicos de Pseudomonas aerugnosa resistentes a carbapenémicos identificadas genotípicamente como portadoras de MBL (17 cepas: 14 con el gen $b l a_{\mathrm{IMP}}$ y 3 con el gen $b l a_{\mathrm{VIM}}$ ) y no portadoras de MBL (12 cepas) (recolectadas entre 2011 y 2017) del cepario del laboratorio de Epidemiología Molecular y Genética del Instituto de Medicina Tropical Daniel A. Carrión de la Universidad Nacional Mayor de San Marcos. Luego fueron transportados al laboratorio de Patología Clínica del Hospital de Alta Complejidad Virgen de la Puerta, EsSalud, donde se almacenaron a $-20^{\circ} \mathrm{C}$ hasta su procesamiento.

Se reactivaron las cepas, evaluando su pureza y viabilidad. Luego se realizaron dos suspensiones bacterianas (escala 0,5 McFarland) por aislamiento. A una suspensión bacteriana se incubó con MEM PHARMAGEN ${ }^{\circledR}(8 \mu \mathrm{g} / \mathrm{mL})$; a la segunda suspensión bacteriana se incubó con MEM PHARMAGEN ${ }^{\circledR}$ $(8 \mu \mathrm{g} / \mathrm{mL})$ más el inhibidor EDTA Merk ${ }^{\circledast}(12,5 \mathrm{mM})$. Ambas suspensiones se incubaron a $37^{\circ} \mathrm{C}$ por dos horas ${ }^{(11)}$.

Después de dos horas de incubación se procedió a realizar el test de viabilidad con el kit de viabilidad celular de Becton 
Dickinson (BD). Se agregó el reactivo del kit de viabilidad celular $(5 \mu \mathrm{L}$ de Tiazol Orange [TO], $42 \mu \mathrm{mol} / \mathrm{L}$ en dimetilsulfoxido y $5 \mu \mathrm{L}$ de yoduro de propidio [IP] $4,3 \mathrm{mmol} / \mathrm{L}$ en agua) a $500 \mu \mathrm{L}$ de la suspensión bacteriana previamente tratada. Se mezcló e incubó por 5 minutos a temperatura ambiente en campo oscuro. Los voltajes se ajustaron de manera gráfica para diferenciar la población bacteriana marcada con TO, marcador de celularidad, de los debris (restos celulares).

Se realizó la lectura en el citómetro de flujo FACSCalibur $\mathrm{BD}$. Se interpretaron los resultados en función del aumento o no de fluorescencia emitida por el IP, siendo positivo a detección de MBL si hay aumento significativo en la razón de fluorescencia de la suspensión tratada con MEM más el EDTA en comparación con la suspensión incubada solo con MEM. Todo el procedimiento fue acompañado por tubo testigo donde solamente se incubó la suspensión bacteriana de cada cepa, sin antibiótico ni inhibidor y se trató con el kit de Viabilidad Celular (BD) para confirmar la viabilidad de las bacterias frente a los reactivos utilizados.

Las muestras se analizaron con el programa Cell Quest', donde se clasificaron las bacterias según la fluorescencia emitida, cuantificándola como «evento». Si un evento es positivo solo a TO, se clasificará en la categoría de «vivas»; si es positivo a IP y TO, se clasificarán como «dañadas», y si es positivo solo a IP se clasificarán como «degradadas». A fin de obtener una cantidad medible y comparable, con el porcentaje de eventos obtenidos se comparó el índice de aumento de eventos obtenidos entre el tubo con MEM-EDTA y el el tubo con MEM (\% eventos tubo con MEM-EDTA / \% eventos tubo con MEM), con los aislamientos productores de MBL y con los no productores de MBL.

Se realizó la prueba de T de student, se elaboró el análisis de curva ROC, se determinó la sensibilidad y especificidad. Se utilizó el programa estadístico SPSS 24.

El Comité de Ética de la Universidad Nacional de Trujillo aprobó el protocolo del estudio. Todos los procedimientos del presente estudio se realizaron de acuerdo con los lineamientos de las buenas prácticas clínicas y de ética en investigación biomédica.

\section{HALLAZGOS}

Mediante CF, usando el kit de viabilidad celular, se identificaron $16 P$. aeruginosa como productoras de MBL y $13 P$. aeruginosa como no productoras de MBL. Al comparar el índice de aumento de fluorescencia entre las células «no vivas», siendo esta la suma del porcentaje de eventos entre las células clasificadas como «dañadas» y las «degradadas»; en los tubos con el antibiótico más el inhibidor en comparación con el tubo solo con antibiótico, se obtuvo que el índice promedio en las $P$. aeruginosa productoras de MBL fue de 3,17 y de 1,09 para las no productoras de MBL, lo cual arroja una diferencia significativa $(p<0,01)$ (Figura 1$)$.

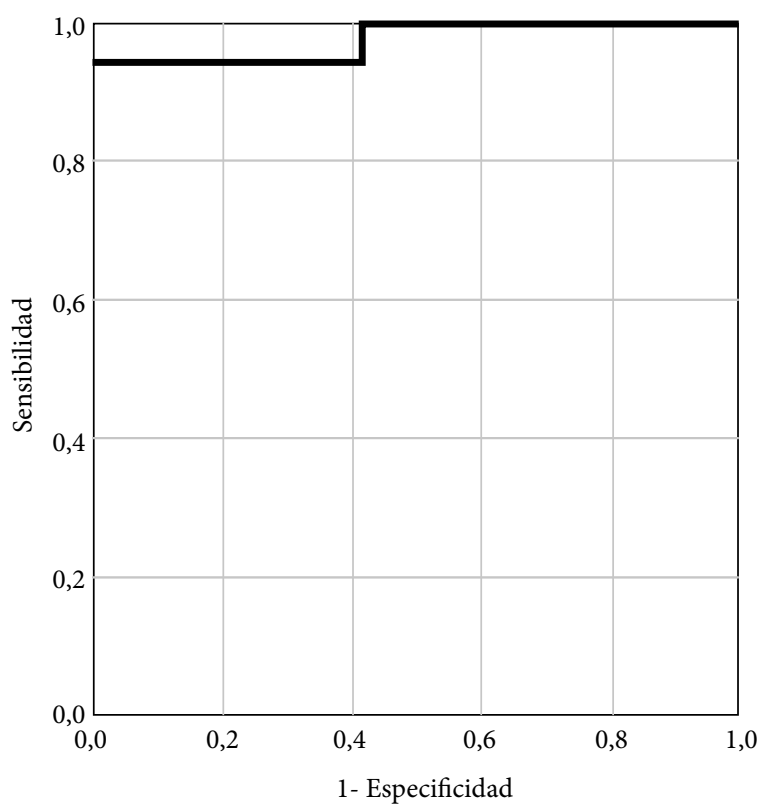

Área bajo la curva (ABC): 0,975

Figura 1. Curva ROC del análisis por citometría de flujo para la detección de metalobetalactamasas

Para evaluar la sensibilidad y especificidad del análisis por $\mathrm{CF}$ para la detección de $\mathrm{MBL}$, se realizó un análisis mediante curva ROC y se encontró un área bajo la curva $(\mathrm{ABC})$ de 0,975 (Figura 1), lo que sugiere como mejor punto de corte 1,6 para el índice de células «no vivas».

Con los resultados genotípicos (prueba de oro) y los resultados obtenidos por citometría de flujo, usando el punto de corte de 1,6 en el índice de aumento de células «no vivas», se obtuvo una sensibilidad de $94,1 \%$ y una especificidad del $100 \%$; además, un valor predictivo positivo (VPP) de 100\% (Figura 2).

\section{DISCUSIÓN}

En esta investigación presentamos un método alternativo para la detección de MBL en $P$. aeruginosa usando la CF con el kit de viabilidad celular BD, evaluando la cepa bacteriana sola, la cepa frente al antibiótico carbapenémico (MEM) y la cepa frente al antibiótico más el inhibidor de MBL (EDTA), observándose resultados satisfactorios, con una sensibilidad de $94,1 \%$ y una especificidad de $100 \%$.

Estudios como el de Mulroney et al. ${ }^{(12)} \mathrm{y}$ el de Kilic et al. ${ }^{(13)}$ han utilizado la CF como predictor de la resistencia a los carbapenémicos en solo dos horas. Sin embargo, no diferenciaron la presencia o no de carbapenemasas. El estudio de Pina-Vaz et al. ${ }^{(14)}$ combina la $\mathrm{CF}$ con el análisis computacional para la detección de carbapenemasas de clase $\mathrm{A}, \mathrm{B}$ y $\mathrm{D}$ en un número pequeño de aislamientos, sin reportar sensibilidad ni especificidad. Silva et al. ${ }^{(11)}$, usando CF para la detección de carbapenemasas en enterobacterias (30 PC y 30 No-PC), reportó un 100\% de sensibilidad y especificidad. Esto es similar a los resultados 


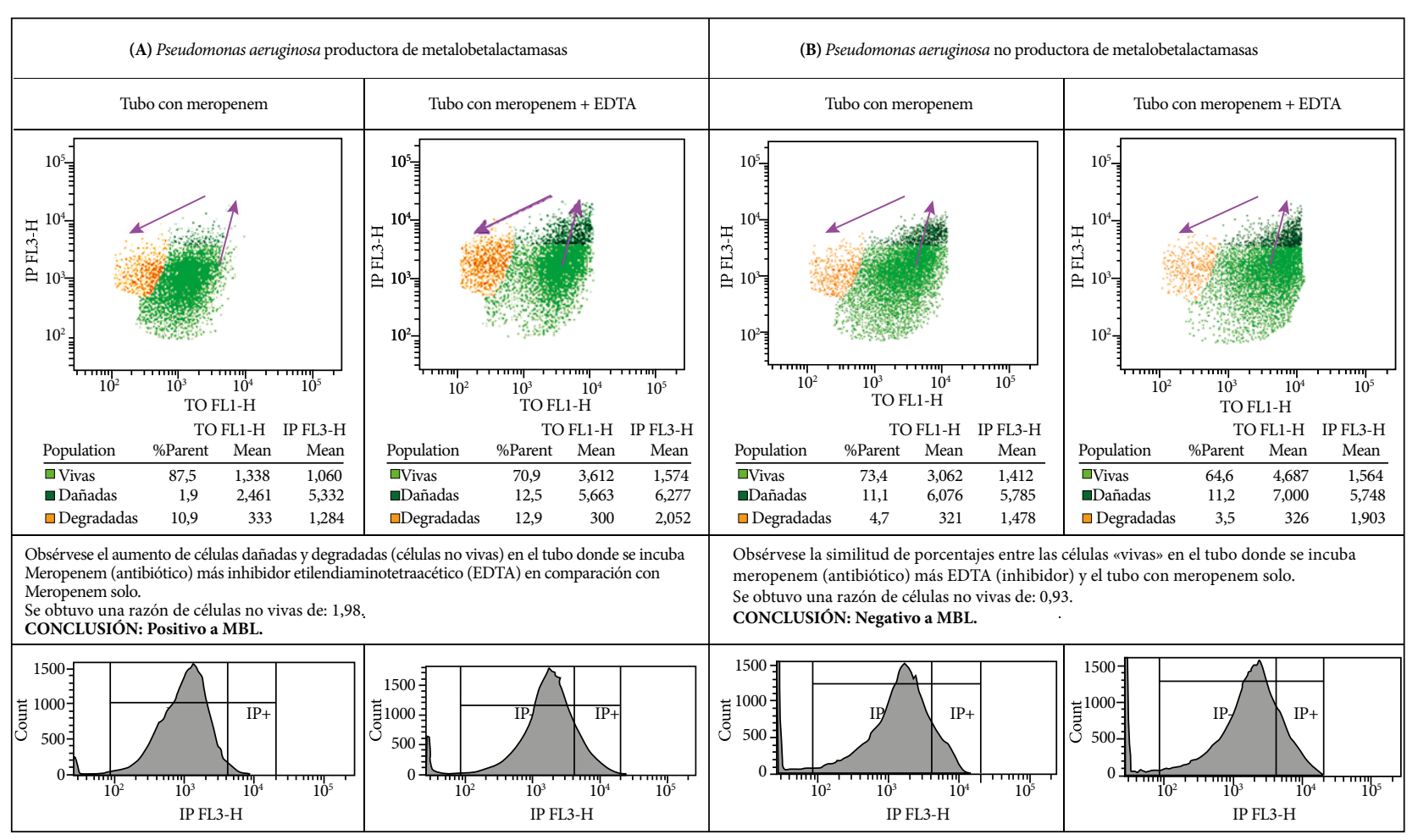

Figura 2. Análisis mediante citometría de flujo para la detección de $P$. aeruginosa productoras o no de metalobetalactamasas.

obtenidos en nuestra investigación. La diferencia en la sensibilidad pudo deberse a que se trabajó con un grupo bacteriano distinto y a que solo una cepa (1/17 MBL) de P. aeruginosa portadora del gen $\mathrm{bla}_{\mathrm{IMP}}$ no fue detectada por la $\mathrm{CF}$, considerándose un falso negativo; esto se debe posiblemente a una baja expresión de este tipo de enzimas de MBL en la cepa estudiada.

Entre otros estudios que usan la CF para la detección de betalactamasas está el realizado por Faria-Ramos et al. ${ }^{(15)}$, donde usó el citómetro de flujo FACSCalibur para buscar betalactamasas de espectro extendido (BLEE) en enterobacterias, con un índice de aumento de fluorescencia de 1,5 entre el antibiótico más el inhibidor (ácido clavulánico) y el antibiótico solo, índice semejante al reportado en esta investigación. Por otra parte, Akhmaltdinova ${ }^{(16)}$ comparó microdilución y la CF para la detección de BLEE, siendo el primero un método de referencia, y reportó una sensibilidad $85,7 \%$ y especificidad $88,8 \%$.

Dentro de las pruebas fenotípicas de detección de MBL, tenemos el estudio de Heinrichs et al. ${ }^{(7)}$, que compara diversos métodos fenotípicos en 183 aislamientos de $P$. aeruginosa, usando disco de difusión combinado con imipenem $(10 \mu \mathrm{g})+$ EDTA $(750 \mu \mathrm{g})$, y reportó una sensibilidad de 90\% y una especificidad de $93 \%$ y con meropenem $(10 \mu \mathrm{g})+$ DPA $(1000 \mu \mathrm{g})$ obtuvo una sensibilidad de $97 \%$ y una especificidad de $88 \%$. Buscando sinergia, con DPA + imipenem $10 \mu \mathrm{g} /$ meropenem $10 \mu \mathrm{g}$ se obtuvo una sensibilidad y especificidad de $100 \%$ y $31 \%$, respectivamente. Al evaluar la sensibilidad y especificidad de métodos colorimétricos como el CarbaNP reportaron un $88 \%$ y $99 \%$, respectivamente.

Es por esto, que la CF no deja de ser ventajosa frente a los métodos basados en cultivo que deben esperar 24 horas para su interpretación. La CF nos puede proporcionar un resultado previo en dos horas mientras se confirma el hallazgo en las próximas 24 horas. Otro punto importante es que la CF evita la interpretación subjetiva al observar el cambio de color, lo que sucede con las pruebas rápidas colorimétricas, lo que la convierte en una herramienta con la que podemos alcanzar un análisis objetivo mediante estimación de la fluorescencia usando índices, mientras se cuente con un equipo de CF podría fácilmente convertirse en una herramienta orientativa para la búsqueda de MBL en $P$. aeruginosa en corto tiempo.

Dentro de las limitaciones de este trabajo está la cantidad limitada de muestras, debido a que eran necesarios aislamientos confirmados genotípicamente. Futuros trabajos con múltiples mecanismos de resistencia y expresión simultánea (serin carbapenemasas y BLEE) podrían evaluarse para determinar la especificidad para MBL usando este protocolo para $P$. aeruginosa, aunque debemos considerar que la producción de MBL es frecuente en este microorganismo.

En conclusión, nuestros resultados sugieren que los ensayos de CF, evaluando la viabilidad de células con antibiótico y antibiótico más inhibidor, podrían proporcionar un método rápido y de fácil interpretación para detectar $P$. aerugno- 
sa productora de MBL de manera presuntiva. Sin embargo, son necesarios estudios adicionales con un mayor número de aislamientos para confirmar la precisión de esta metodología.

Contribuciones de autoría: Todos los autores han participado en la idea de la investigación, concepción del artículo, la recolección de datos, material de estudio y redacción del artículo. Todos los autores aprobaron la versión final del manuscrito.

Financiamiento: Autofinanciado.

\section{REFERENCIAS BIBLIOGRÁFICAS}

1. Hong DJ, Bae IK, Jang IH, Jeong SH, Kang HK, Lee K. Epidemiology and characteristics of Metallo-ß-Lactamase-producing Pseudomonas aeruginosa. Infect Chemother. 2015;47(2):81-97. doi: 10.3947/ ic.2015.47.2.81.

2. He Q, Chen W, Huang L, Lin Q, Zhang J, Lui R, et al. Perfomance evaluation of three automated identification systems in detecting carbapenem-resistant Enterobacteriaceae. Ann Clin Microbiol Antimicrob. 2016;15(1):40. doi: 10.1186/s12941-016-0154-0.

3. Nordmann P, Poirel L, Dortet L. Rapid detection of carbapenemse-producing Enterobacteriaceae. Emerg Infect Dis. 2012;18(9):1503-7. doi: 10.3201/eid1809.120355.

4. Banerjee R, Humphries R. Clinical and laboratory considerations for rapid detection of carbapenem-resistant Enterobacteriaceae. Virulence. 2017;8(4):427-439. doi: 10.1080/21505594.2016.1185577.

5. Sacha P, Wieczorek P, Hauschild T, Zórawski M, Olszanska D, Tryniszewska E. Metallo- $\beta$-lactamases of Pseudomonas aeruginosa a novel mechanism resistance to $\beta$-lactam antibiotics. Folia Histochem Cytobiol. 2008;46(2):137-42. doi: 10.2478/v10042-008-0020-9.

6. Kumar MS, Lakshmi V, Rajagopalan R. Ocurrence of extended spectrum $\beta$-lactamases among Enterobacteriaceae spp. Isolated at a tertiary care institute. Indian J Med Microbiol. 2006;24(3):208-11.

7. Gonzales-Escalante E, Vicente-Taboada W, Champi-Merino R, Soto-Pastrana J, Flores-Paredes W, Lovera-García M, et al. Metalo-B-lactamasas en aislamientos clínicos de Pseudomonas aeruginosa en Lima, Perú. Rev Peru Med Exp Salud Publica. 2013;30(2):241-5.

8. Salvador-Luján G, García-de-la-Guarda R, Gonzales-Escalante E. Caracterización de metalo- $\beta$-lactamasas en aislados clínicos de Pseudomonas aeruginosa recuperados de pacientes hospitalizados en el Hospital Militar Central. Rev Peru Med Exp Salud Publica. 2018;35(4):636-641. doi: 10.17843/rpmesp.2018.354.3755.
Conflictos de interés: Los autores declaran no tener conflicto de interés.

Agradecimientos: Al personal del Laboratorio del Hospital de Alta Complejidad Virgen de la Puerta por el apoyo, al laboratorio de Epidemiología Molecular y Genética del Instituto de Medicina Tropical Daniel A. Carrión UNMSM por facilitar las cepas para la realización del estudio, a la Dra. Patricia Contreras y al Mg. Paul Ríos Sanca por la colaboración para la realización de esta investigación.

9. Heinrichs A, Huang TD, Berhin C, Boagerts P, Glupczynski Y. Evaluation of several phenotypic methods for the detection of carbapenemse-producing Pseudomonas aeruginosa. Eur J Clin Microbiol Infect Dis. 2015;34(7):1467-74. doi: 10.1007/s10096-015-2376-z.

10. Díaz M, Herrero M, García AL, Quirós C. Application of flow cytometry to industrial microbial bioprocesses. Biochem Eng J. 2010; 48(3):385-407. doi: 10.1016/j.bej.2009.07.013

11. Silva A, Faria-Ramos I, Ricardo E, Miranda MI, Espinar MJ, Costa-de-Oliveira $\mathrm{S}$, et al. Rapid flow cytometry test for identification of different carbapenemses in Enterobacteriaceae. Antimicrob Agents Chemother. 2016;60(6):3824-6. doi: 10.1128/AAC.02947-15.

12. Mulroney KT, Hall JM, Huang X, Turnbull E, Bzdyl NM, Chakera A, et al. Rapid susceptibility profiling of carbapenem-resistant Klebsiella pneumoniae. Sci Rep. 2017;7(1):1903. doi: 10.1038/s41598-017-02009-3.

13. Kilic A, Dogan E, Kaya S, Oren S, Tok D, Ardic N, et al. Rapid Identification of Klebsiella pneumoniae by Matrix-Assisted Laser Desorption/Ionization-Time of Flight Mass Spectrometry and Detection of Meropenem Resistance by Flow Cytometric Assay. J Clin Lab Anal. 2016;30(6):11911197. doi: 10.1002/jcla.22002.

14. Pina-Vaz C, Silva AP, Faria-Ramos I, Teixeira-Santos R, Moura D, Vieira TF, et al. A Flow Cytometric and Computational Approaches to Carbapenems Affinity to the Different Types of Carbapenemases. Front Microbiol. 2016; 7: 1259. doi: 10.3389/fmicb.2016.01259.

15. Faria-Ramos I, Espinar MJ, Rocha R, Santos-Antunes J, Rodrigues AG, Cantón R, et al. A novel flow cytometric assay for rapid detection of extended-spectrum $\beta$-lactamases. Clin Microbiol Infect. 2013;19(1):E8-E15. doi: 10.1111/j.1469-0691.2012.03986.x.

16. Akhmaltdinova L, Lavrinenko A, Belyayev I. Flow Cytometry in Detecting Resistant E. coli Strains. Open Access Maced J Med Sci. 2017 Jul 28;5(5):592-594. doi: 10.3889/oamjms.2017.104. 